FORMATION Formation emploi

Revue française de sciences sociales

97 | janvier-mars 2007

Former pour dynamiser les territoires

\title{
Bibliographie sélective sur le thème « la formation et les territoires »
}

\section{Marie Baudry de Vaux}

\section{(2) OpenEdition}

1 Journals

\section{Édition électronique}

URL : http://journals.openedition.org/formationemploi/1712

DOI : 10.4000/formationemploi.1712

ISSN : 2107-0946

\section{Éditeur}

La Documentation française

\section{Édition imprimée}

Date de publication : 1 janvier 2007

Pagination : 89-93

ISSN : 0759-6340

\section{Référence électronique}

Marie Baudry de Vaux, "Bibliographie sélective sur le thème «la formation et les territoires » »,

Formation emploi [En ligne], 97 | janvier-mars 2007, mis en ligne le 19 février 2009, consulté le 30 octobre 2020. URL : http://journals.openedition.org/formationemploi/1712 ; DOI : https://doi.org/ 10.4000/formationemploi.1712 


\title{
Bibliographie
}

\author{
Bibliographie sélective sur le thème "la formation et les territoires". \\ Les références ont été sélectionnées à partir de l'année 2000. \\ Elles sont classées par ordre alphabétique d'auteur. \\ Les résumés proviennent de la base documentaire du Céreq. \\ Cette bibliographie a été réalisée par Marie Baudry de Vaux.
}

\section{Territoires en formation}

Formation Emploi, octobre-décembre 2003, n 84 , pp. 5-87

Ces territoires qui façonnent l'insertion Formation Emploi, juillet-septembre 2004, n 87 , pp. 1-124

\section{Formation professionnelle.}

Regards sur les politiques régionales

Baunay Yves, Vergne Francis

Paris, Éditions Nouveaux regards/Syllepse, 2006, $161 \mathrm{p}$.

Collection «Comprendre et agir »

\section{Décentralisation de la formation professionnelle : un processus en voie d'achèvement?}

Bel Maïten (Dir.), Dubouchet Louis (Dir.) La Tour d'Aigues, Éditions de l'Aube, 2004, 153 p. Collection "Bibliothèque des territoires »

Comment s'inscrit réellement le processus de décentralisation de la formation professionnelle de l'État vers les régions, lors de la mise en place des dernières mesures législatives? Par ailleurs, la formation professionnelle constitue dans les régions un des postes les plus importants des dépenses.
S'appuyant sur ces deux éléments, les auteurs dressent un bilan, depuis les années 80, de la façon dont les régions se sont emparées de cette responsabilité de mise en cohérence de l'offre de formation professionnelle sur leur territoire. Mais de quoi s'agit-il exactement? La décentralisation de la formation est l'opération par laquelle l'État transfère aux collectivités territoriales des compétences précédemment détenues par une administration centrale telles la construction et la validation des diplômes, le financement des équipements ou encore la gestion des personnels de formation. Un premier chapitre constate une forte hétérogénéité de l'offre de formation sur le territoire et montre toute la difficulté de mesurer l'impact des politiques régionales. Le second est consacré au bilan des vingt cinq dernières années en termes d'évolution des institutions régionales et de construction d'un pouvoir régional. L'inscription dans l'espace du territoire défini par l'État est déterminante dans l'action menée par les conseils régionaux. Autrement dit, quel est l'effet sur le développement des territoires des politiques régionales de formation professionnelle? Le rôle de " chef de file» attribué aux conseils régionaux est analysé afin de comprendre la façon dont ils assument cette nouvelle responsabilité de mettre en cohérence, au niveau local, l'offre proposée et l'équilibre des territoires. Enfin, un dernier chapitre, en s'appuyant sur une région exemplaire, permet d'analyser ces transformations depuis la mise en œuvre de la loi quinquennale. 


\section{Lad}

La decentralisation

de la formation professionnelle: quels changements dans la conduite de l'action publique?

Bel Maïten (Coord.), Méhaut Philippe (Coord.), Mériaux Olivier (Coord.)

Paris, L'Harmattan, 2003, 252 p.

Collection "Logiques politiques »

Les contributions rassemblées dans cet ouvrage sont issues d'un colloque organisé en 2001 à Grenoble par le Céreq et le CERAT (Centre de recherches sur l'administration, la politique et le territoire - CNRS). Elles ont puisé leurs matériaux dans les travaux d'évaluation menés par le Comité de coordination de la formation professionnelle et de l'apprentissage de 1994 à 2000. Elles proposent des éclairages comparatifs avec d'autres pays européens (Belgique, Allemagne), ou avec des segments de l'action publique (santé, enseignement supérieur) où la combinatoire national/région s'exprime différemment. Elles ont enfin des références disciplinaires variées : économie ou sociologie des politiques publiques, sciences politiques, dont la confrontation autour de la décentralisation était l'un des objectifs de ce colloque. Elles sont organisées en trois chapitres. Le premier est consacré à l'analyse de la décentralisation à l'aune des spécificités nationales ou régionales. Le deuxième aborde la question des recompositions des politiques publiques. Le troisième enfin, traite des outils d'analyse et de conduite de ces politiques.

\section{Les organismes}

paritaires collecteurs agréés.

Acteurs du changement des comportements de formation des petites entreprises

Bentabet Elyes (Coord.), Théry Michel (Coord.)
Marseille, Céreq, 2005, 108 p.
Collection « Relief n 1 1, série Rapports du Céreq »

Après avoir analysé l'impact de la loi quinquennale sur les pratiques des organismes paritaires collecteurs agréés (OPCA), le Céreq a souhaité examiner le rôle de ces derniers dans le développement de la formation professionnelle des petites entreprises. Il publie aujourd'hui les résultats de cette étude qui s'appuie sur plusieurs types d'approches : des recueils documentaires, des entretiens et des questionnaires. Ces résultats s'articulent autour de quatre grands axes : les stratégies des OPCA en direction des entreprises de moins de 50 salariés, les relations de proximité que les OPCA entretiennent avec les petites entreprises au niveau local, la professionnalité des salariés des OPCA, les particularités du financement de la FPC en France.

\section{Des emplois près de chez vous? : la territorialisation des politiques $d^{\prime}$ 'emploi en questions}

\author{
Berthet Thierry (Dir.) \\ Bordeaux, Presses universitaires de Bordeaux, \\ 2005, $226 \mathrm{p}$ \\ Collection «Le territoire du politique. Politiques du \\ social »
}

Depuis vingt ans, la lutte contre le chômage est l'un des objectifs de l'État providence. Les gouvernements ont cherché à prendre en compte les particularités des territoires pour ajuster au mieux la politique de l'emploi aux réalités des marchés locaux du travail. Quelles sont les conséquences de cette territorialisation de l'action publique et quel est le rôle des acteurs locaux en matière d'emploi ? En étudiant la notion de territoire puis sa relation depuis trente ans avec les politiques d'emploi, les chercheurs montrent les paradoxes qui peuvent apparaître quand on définit un intérêt général localisé suite à la décentralisation des politiques publiques en faveur de l'emploi. Dans un deuxième temps, ils mettent en lumière les problèmes soulevés par cette territorialisation des politiques de l'emploi. Cette dernière nécessite la mise en place de partenariats entre les milieux économiques, les acteurs locaux et l'administration de l'emploi pour la mise en œuvre des mesures d'aide. Une dernière réflexion illustre cette question des politiques locales de l'emploi au regard de l'égalité entre les hommes et les femmes.

Villes et développement éducatif local : les cas d'Evry, d'Amiens et de Calais

Chambon André

Paris, Éditions L'Harmattan, 2004, 232 p.

Collection «Savoir et formation» 
Évaluation des politiques régionales de formation professionnelle initiale et continue. 2000-2002

Chevreul Philippe (Prés.)

Paris, Comité de coordination des programmes régionaux d'apprentissage et de formation professionnelle continue, 2004, 274 p.

Ce document rassemble en un seul volume et un cédérom l'ensemble des travaux publiés issus de ce troisième exercice d'évaluation 2000-2002 :

- Le rapport d'évaluation des politiques régionales de formation professionnelle initiale et continue adopté par le Comité de coordination le 14 janvier 2004 ;

- L'étude thématique relative à la coopération et la concertation en région entre les acteurs publics et les acteurs économiques et sociaux réalisée par EUREVAL-C3E ;

- La note de synthèse sur la mise en regard des différents travaux quantitatifs réalisée par le CEREQ ;

- Les typologies et spécificités régionales (CEREQ, THEMA) ;

- Les portraits statistiques réalisés par le CEREQ.

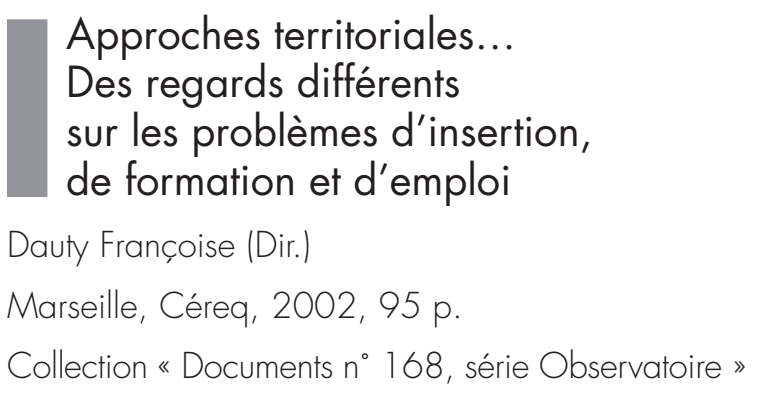

Cet ouvrage présente trois contributions issues des travaux menés dans le cadre d'un groupe de travail « insertion et territoire» composé de membres de centres associés au Céreq, du Céreq et de la DPD, réunis autour des préoccupations relatives aux outils de connaissance et méthodologies mobilisés lorsqu'on aborde les relations formation-emploi-insertion à un niveau décentralisé. Les analyses ont pris appui sur trois questions posées de façon récurrente par les responsables en charge des politiques territoriales :

- Comment instruire des demandes d'ouverturefermeture de sections, d'établissements?
- Quelle définition donner aux notions de publics de «bas niveaux de qualification", de "sortants sans qualification », « de jeunes en difficulté »?

- Les emplois de demain, les gisements d'emploi, les emplois du futur : quels sont-ils?

Au travers des trois contributions aux thématiques $a$ priori assez éloignées apparaissent des éléments communs de réflexion autour de la dynamique des questionnements, de la pertinence des systèmes d'information et des catégories utilisés et de la façon de les mobiliser au regard des différentes logiques d'actions. De manière transversale, l'apport de la dimension territoriale dans les problématiques du champ formation-emploi-insertion est aussi abordé.

Développement des territoires, politiques de l'emploi et formation. L'approche territoriale, un enjeu pour I'AFPA

Denieuil Pierre-Noël, Piriou Odile

Paris, L'Harmattan, 2003, 304 p.

Collection « Logiques sociales »

Les régulations des politiques d'éducation

Dutercq Yves (Dir.)

Rennes, Presses universitaires de Rennes, 2005, 204 p.

Collection «Des sociétés »

La régulation est la recherche de la définition adaptée aux conditions de mise en œuvre du contrôle souple de l'action publique. La décentralisation et la déconcentration des politiques publiques en Europe depuis vingt ans changent les modes de régulation. Les chercheurs, spécialistes de l'action publique en éducation, analysent à partir d'une comparaison internationale les formes de régulation des politiques d'éducation à l'échelon européen, national et local et leurs évolutions. L'analyse de l'ensemble du système éducatif en France permet de mettre en lumière une redistribution récente des pouvoirs locaux et les conséquences des nouvelles missions confiées aux collectivités locales sur le fonctionnement de l'administration déconcentrée de l'Éducation nationale. 
Enfin, une présentation des nouveaux modes de régulation des politiques éducatives en Europe illustre la complexité des imbrications entre les niveaux allant du supranational au micro-local.

\section{L'action publique}

et la question territoriale

Faure Alain (Dir.), Douillet Anne Cécile (Dir.)

Grenoble, PUG, 2005, 300 p.

Collection « Symposium »

Les acteurs politiques et administratifs encouragent, aujourd'hui, la territorialisation de l'action publique au détriment de l'approche sectorielle et professionnalisée. Pour questionner cette montée en puissance des logiques territoriales au cœur des politiques, des chercheurs du Centre de recherches sur l'administration, la politique et le territoire (CERAT), passent au crible les modes de prise en charge des problèmes collectifs (éducation, formation professionnelle...) par les autorités publiques. Puis ils présentent des études qui évoquent l'émergence d'une dynamique territoriale (le patrimoine identitaire, les produits du terroir). Autour de la notion de gouvernance, qui désigne l'extrême diversité des influences qui traversent les décisions politiques, leurs différentes analyses illustrent la nécessité d'une coexistence des approches sectorielle et territoriale dans l'action publique.

\section{Organisations} et systèmes de formation

Garant Michèle (Dir.), Scieur Philippe (Dir.) Bruxelles, De Boeck Université, 2002, 252 p.

Pour traiter du problème des « pratiques de formation et de gestion du changement dans les organisations ", le colloque réuni à Mons en 1997, a choisi de faire appel à un panel varié d'intervenants : commanditaires et prestataires de formation, chercheurs et praticiens. L'ouvrage reprend l'essentiel de leurs contributions en les regroupant en trois parties. La première, intitulée «L'entreprise et son environnement », aborde les relations entre la formation et le développement local et s'interroge sur l'efficacité de la formation dans le développement économique de la région. La deuxième partie traite de l'entreprise et de ses régulations, et insiste sur la place du développement des compétences individuelles et collectives, dans la réussite de l'entreprise. La dernière partie, axée sur l'«Analyse de l'entreprise qui forme », s'intéresse aux enjeux de la formation dans les organisations, avec notamment une étude sur les relations entre les gestionnaires de PME et les consultants en formation. La conclusion souligne une triple incertitude: incertitude concernant l'intégration de certaines catégories sociales, incertitude relative à « l'horizontalisation » des procédures et des communications et incertitude enfin liée à la décentralisation des structures et de la décision.

\section{Dynamiques de proximité}

Gilly Jean-Pierre (Dir.), Torre André (Dir.)

Paris, L'Harmattan, 2000, 301 p.

Collection «Emploi, industrie et territoire »

\section{Formation et emploi en région. Outils, méthodes, enjeux. Travaux du groupe ORAFE}

Simon-Zarca Georgie (Coord.)

Marseille, Céreq, 2006, 258 p.

La capacité d'expertise technique dans le champ de la formation et de l'emploi est devenue, avec la décentralisation, un enjeu important pour les acteurs régionaux. Alors que les besoins et les attentes vont croissant, de nombreux outils d'analyse ont vu le jour. Cependant, leur diversité rend le paysage de l'expertise régionale formation-emploi relativement opaque pour les non-spécialistes. Quel état des lieux peut-on faire des ressources techniques? Sur quels critères choisir un outil plutôt qu'un autre, pour répondre à quelle question ? D'autre part, le décalage est parfois important entre la demande des décideurs/ utilisateurs qui ont une culture de l'action, et la réponse des concepteurs/techniciens qui ont une culture de la méthode et de l'objectivation du réel. Aux questionnements faussement simples posés par les décideurs, les informations que génèrent les outils apportent-elles des réponses directement mobilisables ? C'est autour de ces questions, de méthode et d'usage des outils en région, qu'un groupe de techniciens et d'experts s'est constitué en séminaire de travail. 
Coordonné par le Céreq, cet ouvrage rend compte de leurs travaux et réflexions.

\section{Innovations institutionnelles et territoires}

Tallard Michèle (Dir.), Théret Bruno (Dir.), Uri Didier (Dir.)

Paris, L'Harmattan, 2000, 449 p.

Collection «Logiques politiques"

Limpact des innovations, qu'elles soient technologiques ou organisationnelles, sur les transformations institutionnelles de l'économie productive, notamment dans les domaines de l'emploi et du travail, a été l'un des principaux thèmes de ce colloque. Les deux autres axes ont porté l'un sur les effets de feedback de l'innovation technologique sur l'innovation institutionnelle caractérisés par des relations de dépendance mutuelle plutôt que sous le seul angle de la contrainte institutionnelle sur les pratiques innovatrices. L'autre axe a concerné les innovations d'échelle, les pratiques innovatrices débordant souvent des cadres territoriaux initiaux. $\mathrm{Ce}$ sont bien les conditions et la façon dont les processus d'innovation s'emparent de l'espace géographique pour créer du territoire, au sens d'espace institutionnalisé, qui forment la trame des contributions rassemblées dans cet ouvrage. Cet ouvrage est issu des travaux d'un colloque organisé par l'IRIS (Institut de recherche interdisciplinaire en socio-économie), en décembre 1998, à l'université Paris-Dauphine, intitulé « Changement institutionnel et dynamique de l'innovation ». 
